\title{
PTU-031 PHYSICAL ACTIVITY AND THE RISK OF DEVELOPING PANCREATIC CANCER - DATA FROM A UK PROSPECTIVE STUDY (EPIC-NORFOLK)
}

doi:10.1136/gut.2011.239301.159

P J R Banim,,$^{*}$ R Luben, ${ }^{2} \mathrm{~K}-\mathrm{T}$ Khaw, ${ }^{2} \mathrm{~N}$ Wareham, ${ }^{3}$ A R Hart ${ }^{1,4}{ }^{1}$ School of Medicine, University of East Anglia, Norwich, UK; ' Institute of Public Health, Cambridge, UK; ${ }^{3}$ MRC Epidemiology, University of Cambridge, Cambridge, UK; ${ }^{4}$ Gastroenterology, Norfolk and Norwich University NHS Trust, Norwich, UK

Introduction Physical activity may reduce the risk of developing pancreatic cancer by preventing weight gain and improving glucose tolerance and insulin sensitivity. Previous epidemiological investigations of physical activity in large cohort studies have reported inconsistent results, although many showed no associations. The aim of this UK prospective cohort study was to investigate physical activity assessed by a questionnaire validated against detailed physiological measurements. Methods A total of 25639 participants (56\% women) aged 40-74 years were recruited into the European Prospective Investigation into Cancer-Norfolk (EPIC-Norfolk). Participants attended a health check at enrolment where weight and height were recorded and they completed a questionnaire, which recorded occupational and recreational physical activity, which had been validated, firstly against energy expenditure (assessed by 4-day heart rate monitoring), and secondly cardiorespiratory fitness (assessed by sub-maximal oxygen uptake). From the questionnaire participants were classified into four groups of physical activity: active, moderately active, moderately inactive and inactive. The cohort was then monitored for 16 years and cancer registry data used to identify those who subsequently developed pancreatic cancer. A cohort analysis was performed using Cox regression modelling to estimate the hazard ratios (HR) for developing pancreatic cancer for each category of physical activity, adjusted for age, gender, smoking status, body mass index and diabetes.

Results A total of 93 participants (52.6\% women, mean age of diagnosis $=72.2$ years $(\mathrm{SD}=9.0$ years) $)$ developed pancreatic cancer with $77 \%$ having advanced disease. The interval between recruitment and diagnosis was 8.6 years ( $\mathrm{SD}=3.7$ years). No associations were found between increased physical activity and pancreatic cancer (active vs inactive $\mathrm{HR}=1.03,95 \% \mathrm{CI} 0.52$ to 2.01 , trend across categories $\mathrm{HR}=1.0495 \%$ CI 0.84 to 1.28 ).

Conclusion Physical activity was not associated with the risk of developing pancreatic cancer in a UK population, a result supported by most previous studies. The evidence is emerging that physical activity is not involved in the aetiology of pancreatic cancer.

Competing interests None.

Keywords Aetiology, Pancreatic cancer, Physical activity. 